\title{
On Line Elaboration of a Mental Model During the Understanding of an Animation
}

\author{
Emmanuel Schneider and Jean-Michel Boucheix \\ LEAD/UMR CNRS 5022, Université de Bourgogne, Pôle AAFE, Esplanade Erasme, \\ BP 26513, 21065 Dijon Cedex, France \\ emmanuel.schneider@leadserv.u-bourgnogne.fr, \\ jean-michel. boucheix@u-bourgogne.fr
}

\begin{abstract}
This experiment examines how, high and low mechanical and spatial abilities, learners understand an animation. Two variables were manipulated: the controllability of the animations and the task type of the learners to study the device. The comprehension test results indicated a positive effect of a fully controllable animation and also a positive effect of task type, when the attention of the learner is focused on the functional model and on local kinematics. The eye tracking data indicated that the learners attend more to the areas of the animations where a great amount of motion is involved along the causal chain of events. We show an effect of the controllability of the system and of the task type of the learner on the amount of eye fixations and on the number of transitions between areas that included the causal chain.
\end{abstract}

\section{Introduction}

Today, the rapid growth of technology produces a large variety of animations in learning and educational areas. In spite of this amazing development, the cognitive benefits of animated illustrations for the better comprehension of technical or scientific documents still remain hypothetical (Bétrancourt \& Tversky, 2000; Tversky, Bauer-Morrison \& Bétrancourt, 2002). Some of the research shows a positive effect of animation in complex system comprehension (Mayer; 2001; Hidrio \& Jamet, 2002; Rieber, Tzeng \& Tribble, 2004; Boucheix \& Schneider, submitted), but much other research shows no effect (Palmiter \& Elkerton, 1993, Rouet, Merlet, Ros, Richard \& Michaud, 2002) or a negative effect (Lowe, 1999; Schnotz \& Grzondziel, 1999 and recently Mayer, Hegarty, Mayer \& Campbell, 2005). Methodological and cognitive explanations have been proposed to explain the contradictions which exist in this large body of results (Tversky, Bauer-Morrison \& Bétrancourt, 2002; Boucheix \& Guignard, 2005). An interesting explanation for the lack of cognitive benefits of animations appear to be the frequent violation of the apprehension principle defined by Tversky, Bauer-Morrison \& Bétrancourt (2002): "the structure and content of the external representation should be readily and accurately perceived and comprehended" (p. 256). From this perspective, attentional regulation appears to be a central notion in the comprehension processes of a complex system. Taking this point of view, an overly realistic behavioural animation does not seem to be the best way to assist the subject during their building of a model of the 
described system. Realism could be the wrong condition of delivery for spatial and temporal changes of an animation.

The experiment presented in this paper tests two possible ways to increase the apprehendability of an animated mechanical system: The first attends to the level controllability of the animation; The second focuses on the orientation of attention by the specific's task.

\subsection{Interactivity and Learner Control of the Animation}

Recently, a number of researchers explored the "principle of interactivity" (Bétrancourt, 2005) by studying learner-control on the animation (Mayer \& Chandler, 2001; Tassini \& Bétrancourt, 2003; Schwan \& Riempp, 2004; Lowe, 2004, Bétrancourt \& Réalini, 2005; Boucheix \& Guignard, 2005; Boucheix \& Schneider, submitted). Among this research, some results show a benefit of interactive presentation in the case of a basic control of the narration and rhythm of the animated lesson (Mayer \& Chandler, 2001; Boucheix \& Guignard, 2005) and in the domain of procedural tasks (for example tying nautical knots, Schwan \& Riempp, 2004). In some cases the benefits of the interactive presentation is limited to the group of participants with low spatial abilities and mechanical reasoning, or with weak previous knowledge (Boucheix \& Schneider, submitted, Boucheix, submitted). However, a high level of interactivity is not always a beneficial method of presentation. Another body of research shows that the use of animation with full interactivity is not effective for the learner with little prior knowledge of complex systems (Lowe, 2004; Bogacz \& Trafton, 2005), or may shows no (or negative) benefits of control on the animation (Tassini \& Bétrancourt, 2004; Bétrancourt \& Réalini, 2005). A fully interactive animation can be source of cognitive problems: the participants may not correctly use all the possibilities of a control during the animation, and the activity of control itself can also produce an added cognitive demand.

\subsection{Attentional Orientation: Organisational or Functional}

Another way to enhance the apprehension of animations could be to focus the orientation of learner's attention on specific information. For example, during the learning of a mechanical system, the subject has to focus his attention on the configuration of the main pieces of the system in order to realize a mental decomposition of the elements (Narayanan \& Hegarty, 1998), in this case the learner lends great importance to the organisation of the diagram. But during the study of the mechanical diagram the subject should also favour the functional dimension of the system: The kinematics and causal chain function of the device. The effect of these two kinds of strategy on the elaboration of an accurate mental model of the system may not be equivalent.

We can hypothesize that as a function of the goal of the task specified to the learner (for example, focused on the organisation of the elements or focused on the functional causal chain of the system), their need for information is not equal. Interactive controllable animations could be more suitable than no-controllable and less apprehendable ones for their to precisely process causal chain and kinematics information. The learner focus of attention on the functional model with in a specific 
task could facilitate the information processing, and the user's control could improve the quality of this processing

In the experimental investigation presented below, we study three different levels of user's control during the learning of a diagram about three pulleys system by adults participants (figure 1). This three pulley system is similar to the famous and wellknown system used by Hegarty (1992), Hegarty \& Just (1993), Hegarty \& Sims (1994), Ferguson \& Hegarty (1995), and Sims \& Hegarty (1997), and also, Hegarty (2004, 2005). The different user's control modalities for the course of dynamic simulation in the functioning of the three pulley system are: First, a no controllable animation; Second, a partial controllable animation, and; Third, a full manipulable one. For each level of control, we specified four different levels of task specification (plus a control one). The goal of this task specification is to influence the orientation of the learner's attention during their study time using the animated system. The three levels of task specification proposed to the participants are related to the different levels of processing of a mechanical device observed by Narayanan \& Hegarty (1998, 2002): The first level concerns the configuration of the main elements of the system; The second level is related to local kinematics (rotation and direction of each pulley) of the system; the last level includes the entire causal chain of the system (Boucheix $\&$ Schneider, submitted). Two groups of subjects are contrasted in the experiment: a group of participants with high spatial abilities with a second group with low spatial abilities.

\subsection{Off Line and One-Line Measures of Cognitive Processing During Comprehension}

Except few pioneers works (for example, Hegarty \& Just, 1989, 1993; Hegarty, 1992) the great majority of research in multimedia comprehension was focused on the animated illustrations used in off line written questionnaires to measure comprehension performance (this situation has begun to change with the growing use of eye tracking apparatus and software). The comprehension questionnaire can measure the comprehension level of the learner, but it is not adequate to understand the dynamic acquisition and integration of information. At this time, we ignore the nature of the on-line cognitive processing of animations. In the experiment presented below we measure the on-line processing of animations of the three pulley system, using an eye tracking technique, followed by off line comprehension measures. The previous research, that make use of eye tracking, has studied mainly text and static diagrams. Eye data about text and static diagrams depicting a simple pulley system show that a diagram contributes to the elaboration of a referential representation of information delivered by text, like a memory aid for the deeper processing of the information that has already been read (Hegarty \& Just, 1989). Another experiment with eye data (Hegarty, 1992) suggests that, with a static diagram, the learner builds, step by step, a piecemeal mental animation of the system uses the causal chain of events to simulate more precisely the motion of the system. The learner begins to inspect local motions of the first, second, and third pulleys to construct meaningful relations between the components. Then, follows a more global inspection, the goal of which is the integration of the relations between all the elements to enable the elaboration of an animated mental model of the causal chain (Hegarty \& Just, 1993, 
Hegarty \& Sims, 1994). We therefore expect that user's control features and specific orientation of the learner attention will lead to different eye tracking patterns.

\subsection{Experimental Overview: Eye Tracking and Animated Diagrams}

In this study, eye movements are captured during the presentation of an animated three pulleys system without text (see figure 1). How an internal mental model is built with an external animated diagram? Do controllability of the animation and task specifications facilitate? Several eye movement indicators are used to answer this question. The first eye data indicator was the number of fixations in different areas of interest (AOI) in the diagram (each pulley, ropes, load) fixed in the function of the casual chain. The second eye data indicator was the number (and orientation) of the transitions between the different AOIs. This indicator could bring data about the information research strategy of the learner and suggest the nature of the comparative and inferential activity during the study time of the system. The last eye data indicator monitored the "scan path" of each subject, which could give precise information about the dynamic cognitive activity of the learner, related to the causal chain functioning of the mechanical system (Baccino, 2004; Hegarty \& Just, 1993). One interest of this study concerns the possibility of comparisons between the off line comprehension performance and the one-line eye tracking data. Are there best strategies of information processing to elaborate an efficient dynamic mental model of the system?

\section{Method}

\subsection{Subjects}

One hundred and twenty one undergraduate students from the University of Burgundy participated.

\subsection{Stimuli and Apparatus}

\subsubsection{Experimental Task Materials}

The material used in this study was adapted from Hegarty's (1992) experiment and consisted of an animated three pulley system (figure 1). This system was chosen because its development is continuous with different states. We supposed that animations could have an effect for this specific type of system. We used three versions related to the three levels of user control. The subjects were presented with a dynamic animation, or a sequential dynamic animation, or finally, an interactive animation that can be totally controlled ${ }^{1}$.

The non-controllable version consisted of an animated diagram of the pulley system. Animation started when the subjects clicked with the mouse in the diagram area. In the sequential dynamic version, the course of this animation was controllable

\footnotetext{
${ }^{1}$ In all versions, the name of all elements of the system was first displayed for sixty seconds (figure 1). During this time, the subject did not control the animation.
} 

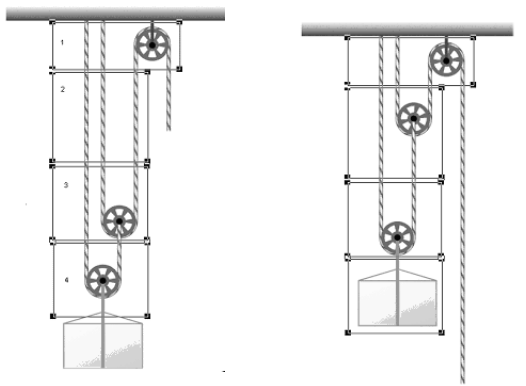

Fig. 1. The three pulleys system no pulled up and pulled up and Areas of interest used to analyze the eye fixations

sequence by sequence (five short animated states) when the subjects clicked with the mouse in the diagram area: one click, one sequence. Subjects could repeat the animation when all the sequences were finished. In the fully controllable version, the learner could manipulate the system, as they wanted by pulling on the end of the free rope, after they have clicked on the end of the high rope. Subjects had total control on the progress of the animation, like an entire simulation of the functioning of the pulleys that lifts the load.

\subsubsection{Memorisation and Comprehension: Off Line Measures}

After the study of the diagram, subjects were asked fourteen memorisation and comprehension questions. Three types of questions were elaborated from the basis of the model of Hegarty $(1992,2004)$ and Narayanan \& Hegarty $(1998,2002)$. The first series of questions concerned the configuration of the elements of the pulleys system (for example: what elements touch the middle pulley?). The second series of questions concerned the local kinematics of the system (rotation and direction of the pulley). The third series of questions concerned the entire functional mental model of the system that involves the integration of all the components and local kinematics. We determined three comprehension sub-scores for each type of questions.

\subsubsection{Individual Differences Measures}

Before the experiment mechanical and spatial abilities of each participant were measured with a French abbreviated form of DAT, Differential Aptitude Tests (Bennett, Seashore \& Wesman, 1973, 2002).

\subsubsection{Apparatus}

The stimulus was presented on a computer screen situated approximately sixty centimetres from the subject. The eye fixations of the subjects were monitored by an ASL (5000) corneal reflectance and pupil-centre eye tracker and were recorded with gaze tracker software, which allowed the processing of dynamic information (like videos, animations, or the internet). Moreover the system was combined with a magnetic head tracker, and therefore this system allowed the subjects to maintain (relatively) free head. ASL 5000 is a 50-hertz system recording the position of the subject's gaze every 20 millisecond. 


\subsection{Procedure}

The overall procedure involved three stages.

During the first stage, the mechanical and spatial abilities were measured collectively with the differential aptitude test. This, then, allowed two groups of participants to be identified: subjects with high and low mechanical and spatial abilities. A mean was calculated for spatial and mechanical scores across all subjects, since we found a significant correlation between the measures $(\mathrm{p}<.01)$. On the base of this single score, we calculated the median score. The median score was 57 percent correct: 60 high (score superior to the median score) and 61 low (score inferior to the median score) mechanical and spatial abilities formed the two contrasted groups. The subjects were then equally distributed among the three type of control and the four level of task specification. Then, five subjects were assigned to each experimental condition. The validity of the distribution was tested with Neuman-Keuls tests.

During the second stage of the experiment, using a computer screen, subjects were presented with one of the three versions (no-controllable, partially controllable and fully controllable) of the animated pulley system. During the study period for the device, eye movements were captured. According to his or her specific task (attention focused on the configuration of the system only, on the local kinematics, on both configuration and overall kinematics of the system, or no specific orientation of attention -control group), the goal assigned to each participant was to understand the operating system. The subjects were informed that they had to use the animation at least three times.

After the consultation of the animated material, subjects answered the comprehension questions test in a booklet.

\section{Results}

In order to stay within the space limits of this paper, the main result about the comprehension performances will be briefly outlined exposed, while the eye tracking data will be discussed in a little bit more detailed.

\subsection{Comprehension Performance}

The mean rates of correct answers picked up at the comprehension test for each indicator (configuration, kinematics, functional model) are presented table 1.

An ANOVA including the three control levels, the two spatial ability levels, the four-task types, and the three dependant comprehension indicators shows firstly a global effect of the spatial abilities. The learner with high spatial and mechanical abilities performed better than the learner with low abilities, $\mathrm{F}(1,97)=21.91$, $\mathrm{p}<$ .0001 . The learner with a fully controllable animated diagram performed slightly better than the learner with no controllable or partial controllable animated diagrams, $\mathrm{F}(2,97)=2.97, \mathrm{p}=.056$. The learners with a specific orientation of attention upon the functional model and, to a lesser extent, with local kinematics performed better than 
the learners with a specific orientation on the configuration elements or with no orientation of attention, $\mathrm{F}(3,97)=4.47, \mathrm{p}=.006$. These effects show that a specific task focuses attention on the functional model and local kinematics allow a better mental model of the system to be constructed. Also, the fully controllable animation allows, to a lesser extent, the building of a better mental model. These results suggest that the elaboration of a mental model can be improved, especially with the orientation specific of attention on a task and also with the user's control of animation.

Moreover, the effect of the comprehension indicators is significant: the scores of configuration are higher than the scores of kinematics which are higher than the scores obtained for the functional model, $\mathrm{F}(2,194)=555.54, \mathrm{p}<.0001$. The interaction between the spatial ability levels and the three indicators of comprehension is significant, $F(2,194)=5.81, p=.004$. This interaction indicates that the learners with high spatial abilities performed better than the learners with low spatial abilities only for the kinematics and functional model questions. The interaction between the task type and the three indicators of comprehension is also significant, $\mathrm{F}(6,194)=5.04, \mathrm{p}=.0008$. This interaction shows a higher benefit when the attention of subjects is focused on the functional model of the system (figure 2). These results have been examined in further detail by three other ANOVAs, one for each comprehension indicator.

For the configuration level, we observed a significant effect for the different orientation of attention, $\mathrm{F}(3,97)=3.47, \mathrm{p}=.019$. The learners with a specific orientation of attention about functional model performed better than the three others specific orientations.

For the kinematics level, we found a significant effect for the spatial ability levels, $\mathrm{F}(1,97)=25.33, \mathrm{p}<.0001$. The learners with high spatial and mechanical abilities performed better than the learners with low abilities for the kinematics questions. Also, we found a significant effect for the level of control, F $(2,97)=4.13, \mathrm{p}=.019$. The learners with a fully controllable animated diagram performed better than the learners with a partially controllable or non controllable animated diagrams. Moreover, we observed an effect of interaction, that approaches significance, between the spatial ability levels and the user control level, $\mathrm{F}(2,97)=2.51, \mathrm{p}=.086$. This interaction shows a weaker performance for the learners with low abilities in particular when the control of the animated diagrams was partial.

Finally, for the functional model level, we observed a significant effect for the spatial ability levels, $\mathrm{F}(1,97)=12.75, \mathrm{p}=.0006$. The learners with high spatial abilities performed better than the learners with low spatial abilities. Moreover, we found a significant effect for the task type, $F(3,97)=7.90, p<.0001$. The learners with a specific orientation of attention upon the functional model or kinematics performed better than the two others specific orientation (configuration and noorientation).

In conclusion, the controllability of the animation allow a better comprehension of the local kinematics and the task type allows better comprehension of the functional model. 
Table 1. Mean rates of correct answers at the three indicators of the comprehension test with each level of control of the pulley animation for each spatial and mechanical aptitude group and for each level of orientation of attention

\begin{tabular}{lccccc}
\hline \multicolumn{3}{c}{ High Spatial } & \multicolumn{3}{c}{ Low spatial } \\
\hline No-control & $\begin{array}{l}\text { Partial } \\
\text { control }\end{array}$ & $\begin{array}{c}\text { Fully } \\
\text { control }\end{array}$ & No-control & Partial & Fully \\
& & & & control & control
\end{tabular}

\begin{tabular}{lcccccc}
\hline $\begin{array}{c}\text { Configuration } \\
\text { score }\end{array}$ & & & & & & \\
No specific task & 79.17 & 81.67 & 90.00 & 80.00 & 73.33 & 75.00 \\
Config. Task & 80.00 & 84.17 & 84.17 & 84.17 & 78.33 & 89.17 \\
Kinematics task & 81.67 & 89.17 & 73.33 & 66.67 & 84.03 & 83.33 \\
Func. model task & 91.67 & 91.67 & 89.17 & 83.33 & 85.83 & 89.17 \\
$\begin{array}{l}\text { Local kinematics } \\
\text { score }\end{array}$ & & & & & & \\
No specific task & 66.90 & 84.14 & 91.72 & 77.93 & 68.67 & 71.03 \\
Config. Task & 74.48 & 71.72 & 88.28 & 69.66 & 47.58 & 68.97 \\
Kinematics task & 82.07 & 91.03 & 71.72 & 68.28 & 72.41 & 82.07 \\
$\begin{array}{l}\text { Func. model task } \\
\text { Functional model }\end{array}$ & 84.83 & 86.21 & 94.48 & 60.69 & 55.17 & 79.31 \\
$\begin{array}{l}\text { score } \\
\text { No specific task }\end{array}$ & 28.33 & 39.17 & 35.00 & 21.67 & 17.50 & 27.50 \\
Config. Task & 33.33 & 25.00 & 32.50 & 25.00 & 19.17 & 32.50 \\
$\begin{array}{l}\text { Kinematics task } \\
\text { Func. model task }\end{array}$ & 45.83 & 45.83 & 40.83 & 39.17 & 37.50 & 43.33 \\
\hline & & 46.67 & 65.00 & 37.50 & 25.00 & 34.17 \\
\hline
\end{tabular}

\subsection{Eye Tracking Measures}

In order to assess the on-line processes in the comprehension of animation, we analyzed the eye fixations of one hundred and thirteen subjects. Areas of interest (AOI) are used to record the amount of time, and activity, the subject's gaze spends in a particular region of the image. We analysed the number of fixations in every AOI, the transition between the different AOI, and the scan path. So, we created four specific AOI, marked in figure 1 . The first AOI included the upper pulley (the only pulley that doesn't move). This AOI correspond to the start time of the causal chain of the system. The second AOI included the middle pulley when this pulley was pulled up during the animated simulation phase. The third AOI included the middle pulley 
before the animation was pulled up and the lower during and after the simulation. In this way, the third AOI was an important area for the integration of the kinematics of the system: the direction and the rotation of two pulleys. In this area we expect a great number of eye movements (comparison transitions). These three AOI characterize the causal chain areas. Finally, the fourth AOI included the lower pulley before the starting of the animation and the area of the loading object during and after the simulation. This AOI seems less relevant for the integration of the kinematics than AOI two and three.

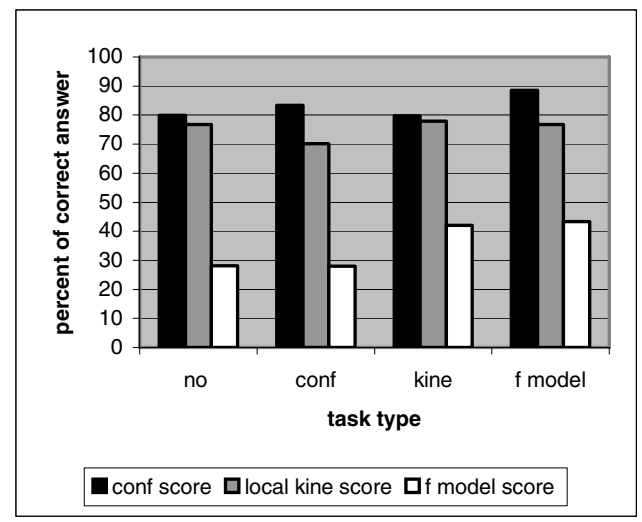

Fig. 2. Percent of correct response for the three comprehension indicators according to the task type

\subsubsection{Number of Fixations}

The number of fixations in the different AOI was the first eye movements measure to be analyzed. The results are displayed in the figures 3, 4. An ANOVA including the three control levels, the two spatial ability levels, the four orientation of attention, and the four AOI as dependant measures shows a significant effect of the control level, $\mathrm{F}(2,89)=7.08, \mathrm{p}=.001$. The number of fixations was more important in the partial control animation than for the full control animation, that was in turn more important than for the no controllable animation. Moreover, the effect of the type of AOI was significant, the number of fixations for the third AOI (area important motions) was higher than the first and second AOI (these two last AOI had a equivalent number of fixations) and higher than the fourth AOI, F $(3,267)=49, \mathrm{p}<.0001$.

In order to go deeper into these results, we conducted four further ANOVAs, one for each AOI. For AOI 1, the partial controllable and the full controllable animation lead to a higher number of fixation than the no controllable animation, $F(2,89)=$ $4.34, \mathrm{p}=.016$. With the same tendency, we observe an effect of the control level for AOI $2, F(2,89)=8.30, p<.001$ and for AOI 3, F $(2,89)=3.78, p=.027$. When the animation could be controlled, the learners watched the third AOI the most, which is more relevant in the understanding the causal chain of the system. During the course of the animation, these AOI deliver a great amount of crucial information about the kinematics of the pulleys. These effects indicates that the number of fixations in the 
partial control level and in the full control level were higher than the no control level for the AOI 1, 2, and 3. But, the number of fixations in the AOI 4 was equal for all of the three levels of control (figure 3 ).

Finally, an interesting result is the fact that, for the AOI 4, which could potentially represent the less pertinent area for the mental integration of the causal chain of the system, we observed a significant effect of the specification of the task, $F(3,89)=2.70$, $\mathrm{p}=05$. The number of fixation on this AOI is higher when there is no specification of the task. This result reveals an interesting fact: the AOI 4 appears less crucial in the processing of the functioning of the three pulleys system, and when the task is specified, the participants spend less time on irrelevant information (cf. figure 4). Moreover, we observe a significant interaction effect between the two spatial ability levels and the four orientations of the attention for this AOI, $\mathrm{F}(3,89)=2.83, \mathrm{p}=.043$. This interaction suggest that the number of fixations in AOI 4 were higher in the condition which no orientation of attention, but only for the subjects with high spatial abilities.

In summary, we observed a number of fixations more important for the AOI that contain a lot of movements and we also observed a number of fixations more important in these AOI for the full and partially controllable animations. Then, we can suppose that attending more to these AOI allows a best integration of the causal chain motions of the system because we have observed better performances for the comprehension test in the two controllable animations conditions. Nevertheless, these results could be also partially due to the activity of control of the device itself. Therefore, in order to allow deeper analysis, this is the reason why we centred the next analysis on the study of the eye transitions between the different AOI for a deeper analyse.

\subsubsection{Transition Between the AOI}

The results about the transitions are presented figures 5, 6. An ANOVA including the three control levels, the two spatial ability levels, the four orientations of attention, and the six possible transitions between the AOI (as dependant measures) shows a

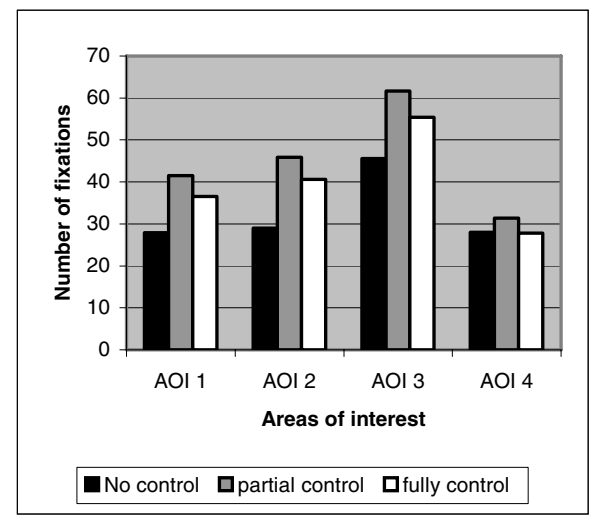

Fig. 3. Number of fixations for every AOI according to the control levels

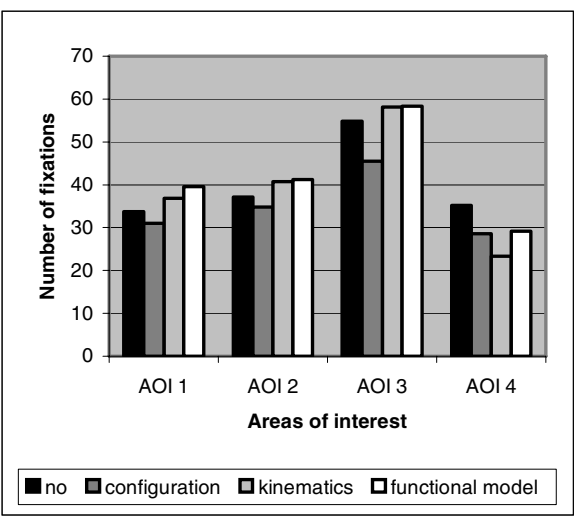

Fig. 4. Number of fixations for every AOI according to the task type 
significant effect of the control level, $\mathrm{F}(2,89)=4.01, \mathrm{p}=.02$. This effect points to the fact that the number of transitions for the partial control level is higher than for the full control level, and the number of transitions for this last level is higher than for the no control level. Moreover, the number of transitions observed between the AOI respectively $1 \& 2,1 \& 3,2 \& 3$, and $3 \& 4$ is higher than the number of transitions observed in the AOI $1 \& 4 ; 2 \& 4, \mathrm{~F}(5,445)=47.12, \mathrm{p}<.0001$. The transitions between AOI $1 \& 2$ and $2 \& 3$ imply the local piecemeal elaboration of the causal chain. The transitions between the look zones $1 \& 3$ suggest a more global integration of the entire functional model of the causal chain of the system. The interaction between the levels of control and the six transitions is significant, $F(10,445)=2.06, p=.026$. This interaction indicates a higher number of transitions between the AOI $1 \& 2,1 \& 3$, and $2 \& 3$ for the controllable levels than for the non controllable one. It can also be observed a few numbers of transitions between the AOI $1 \& 4$, and $2 \& 4$. At last, the number of transitions between the AOI $3 \& 4$ is medium (figure 5).

In order to more precisely examine these broad effects results, we conducted four another ANOVAs, one for each kind of transition area. For AOI 1\&2, the number of transitions for the partial control level is higher than the full control level and the no control level, $\mathrm{F}(2,89)=4.2, \mathrm{p}=.02$. Also we observe a significant effect of the control level for the transition between the AOI $2 \& 3, \mathrm{~F}(2,89)=4.61, \mathrm{p}=.012$. The number of transitions for the controllable conditions was higher than for the non controllable level. Moreover for the areas $2 \& 3$, the results show a significant effect of the attentionnal orientation conditions, $\mathrm{F}(3,89)=2.76, \mathrm{p}=.046$. The number of transitions between the AOI $2 \& 3$, is lower when attention was focused on the system configuration rather than the three others orientation of attention (figure 6).

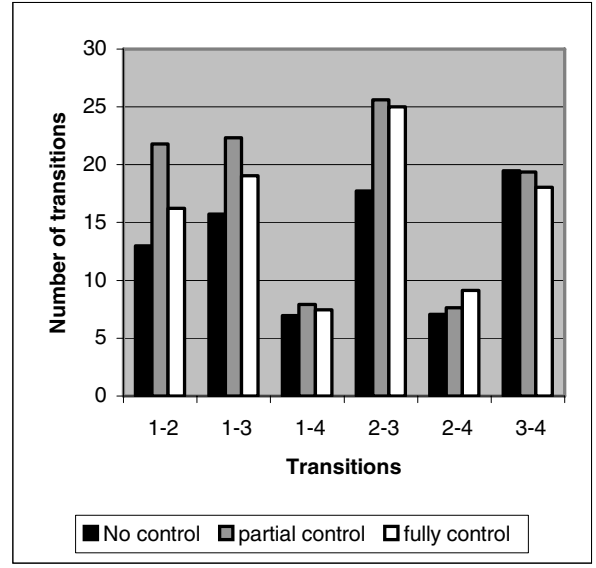

Fig. 5. Number of transitions between the different AOI according to the control levels

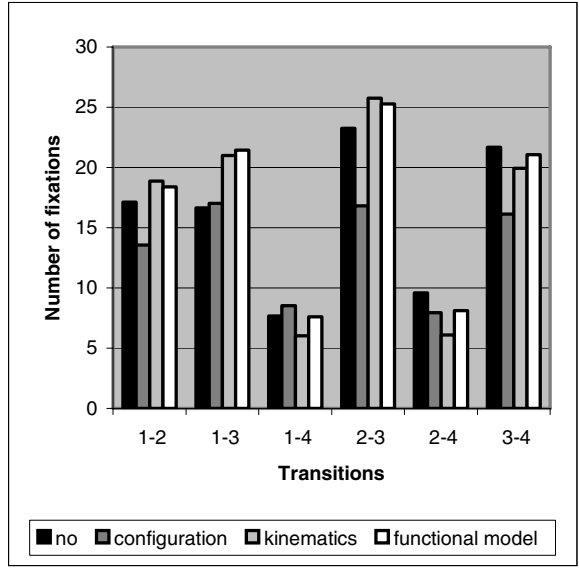

Fig. 6. Number of transitions between the different AOI according to the task type

\subsubsection{Scan Path}

The results previously described can be related to the on line time of processing the three pulleys system during the course of the animation, and for each level of 
controllability and each level of attentionnal orientation. To achieve this goal, the scan path that presents the on line dynamic trajectory of the participant can be used as an essential indicator to visualize the directions of the eye's trajectory and the different strategies used by the learner for the selection of the relevant information. In these proceedings, we will present only the first preliminary results because further analysis is still in progress.

In each learner scan path, we determined three types of transitions between the different look zones. First, we distinguished the global transitions. The global transitions concerned the transitions between two no neighbourhood AOI, for example the transitions between AOI 2 and 4. Second, we distinguished the local transitions. These local transitions concerned the transitions between two neighbourhoods AOI, for example the transitions between AOI 1 and 2. Finally, we distinguished the sequence of transitions (for example, AOI 1,2,3). These "causal chain" transitions concerned the transitions between three neighbourhoods AOI at less, for example the transitions between the AOI 1, 2, 3 or 1, 2, 3, and 4.

The results of these different kinds of transitions (local, global, sequences) are presented in figure 7. An ANOVA including the three control levels, the two spatial ability levels, the four orientations of attention, and the three types of the transitions between the look zones as dependant measures, showing a significant effect of the type of transitions, $\mathrm{F}(2,178)=302.32, \mathrm{p}<.00001$. The number of local transitions is more important than the number of global transitions and this last one is more important than the causal chain transitions. Moreover, results show a significant interaction effect between the different types of AOI and the task type, F(6, 178) = $2.18, \mathrm{p}=.047$. This effect shows that the number of local and causal chain fixations is less important for the configuration task (figure 7).

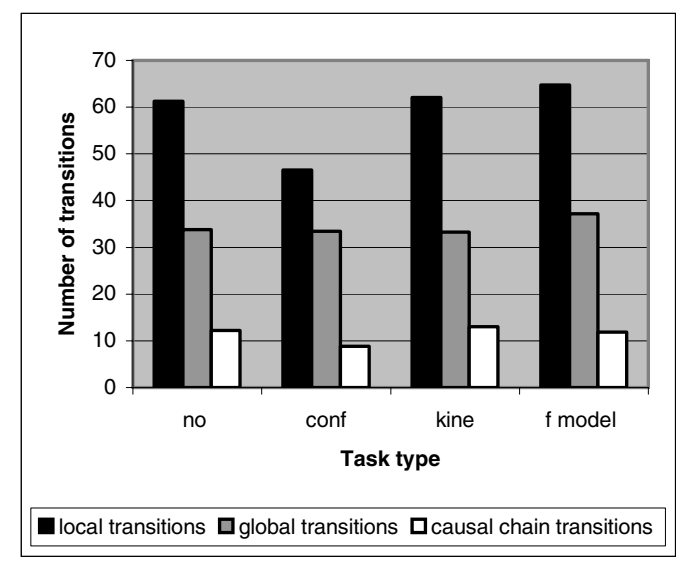

Fig. 7. Number of transitions between the different types of AOI according to the task type

\section{Discussion}

Our goal was to study the effect of a user-controllable animation and of specific orientations of attention in the elaboration of an efficient mental model of a 
mechanical diagram without text. The performances on the comprehension test showed a benefit of the controllable modalities, particularly when the task is oriented toward a mental model level. The full control animation seems to be the relatively most efficient type of control for an effective integration of the mental model of complex mechanical system presented without textual explanation. The orientation of attention by a specific task level showed that the building of the functional mental model was enhanced when the focus of this orientation was focused on the functional model and on the kinematics of the system. The orientation of attention could influence the organization of the integration activity of the different elements and of the relations between the elements according to the causal chain of the system. Although the results showed an effect of the control level and of the specification of the task, they did not show an effect of interaction between these two variables.

The eye tracking measures showed that the number of fixations was more important in the AOI showing the kinematics of the system. The results obtained from the study of the transitions between the different AOI suggest that the learners use the animated features and the controllability of the motions to process the relevant information, which in turn allows them to construct a model of the causal chain of the system. When diagrams are alone, without explanatory texts, interactive animations could enhance significantly the building of an better accurate mental model, and should support best internal simulations. Two sorts of transition were distinguished; transition to integrate local relations, and another to integrate entire functional system. The next analysis, which in progress, of the dynamic processing during the study time of the three pulleys by the scan path, should bring more detailed results about the iterative cycles of the integration: The local, global and sequences of local transitions.

Finally, can our results from the pulley system can be generalized to other complex types of devices or contents? The answer to this question appears to be crucial for the designing of educational systems. We think that the reasoning demands for the comprehension of such pulley system, involving spatial features (rotations, causal chain, speed, direction apprehension) are transferable to other kinds of mechanical systems. Moreover this speculation needs to be experimentally investigated.

\section{References}

Baccino, T. (2004). La lecture électronique: Presses Universitaires de Grenoble.

Bennet, G. K., Seashore, H. G.\& Wesman, A. G. (1973,2002). Differential aptitude test. 5ème edition. ECPA.

Bétrancourt, M. \& Réalini, N. (2005). Le contrôle sur le déroulement de l'animation. $11^{\text {ème }}$ JETCSIC ; 17 Juin, Nice.

Bétrancourt, M. (2005). The animation and interactivity principles in multimedia learning. In R. Mayer (Eds.), The Cambridge handbook of multimedia learning (pp. 287-296). Cambridge: Cambridge University Press.

Bétrancourt, M., \& Tversky, B. (2000). Effect of computer animation on user's performance: a review. Le travail humain, 4, 63, 311-329.

Bogacz, S., \& Trafton, J. G. (2005). Understanding dynamic and static displays: using images to reason dynamically. Cognitive Systems Research, In Press, Corrected Proof.

Boucheix, J.M. (submitted). Children interaction with technical animations: user control and attentionnal cues 
Boucheix, J.M. ; \& Guignard, H. (2005). Which animation condition can improve text comprehension in children? European Journal of Psychology of Education, 20(4), 369-388. .

Boucheix, J.M. ; \& Schneider, E.. (submitted). Designing "apprehendable" animation features to enhance mental animation in multimedia comprehension: micro steps format and user control.

Ferguson, E. L., \& Mary, H. (1995). Learning with real machines or diagrams: application of knowledge to real-word problems. Cognition and Instruction, 13(1), 126-160.

Hegarty, M. (1992). Mental Animation: Inferring Motion From Static Displays of Mechanical Systems. Journal of Experimental Psychology: Learning, Memory, \& Cognition September, 18(5), 1084-1102.

Hegarty, M. (2004). Mechanical reasoning by mental simulation. Trends in Cognitive Sciences, $8(6), 280-285$.

Hegarty, M. (2005). Multimedia learning about physical system. In R. Mayer (Eds.), The Cambridge handbook of multimedia learning (pp. 447-465). Cambridge: Cambridge University Press.

Hegarty, M., \& Just, M. A. (1989). Understanding machines from text and diagrams. In H. Mandl \& J. R. Levin (Eds.), Knowledge acquisition from text and pictures (pp. 171-194). North-Holland: Elsevier Science.

Hegarty, M. \& Just, M.A. (1993). Constructing mental models from texts and diagrams. Journal of Memory and Language, 32, 717-742.

Hegarty, M., \& Sims, V.K. (1994). Individual differences in mental animation during mechanical reasoning. Memory \& Cognition, 22, 411-430.

Hidrio, C., \& Jamet, E. (2002). Compréhension d'un dispositif technique: apports d'une illustration dynamique et des traitements multiples. Psychologie Française, 47-1, 61-67.

Lowe, R.K. (1999). Extracting information from an animation during complex visual learning. European Journal of Psychology of Education. Special Issues: visual Learning, W. Schnotz, (Ed). XIV, 2, 225-244.

Lowe, R.K. (2004). Interrogation of a dynamic visualization during learning. Learning and Instruction, 14, 257-274.

Mayer, R.E. (2001) Multimedia learning. Cambridge: Cambridge University Press.

Mayer, R.E., \& Chandler, P. (2001). When learning is just a clik away: does simple user interaction foster deeper understanding of multimedia messages. Journal of Educational Psychology, 93, 390-397.

Narayanan, N.H., \& Hegarty, M. (1998). On designing comprehensible interactive hypermedia manuals. International Journal of Human-Computer Studies, 48, 267-301.

Narayanan, H. N., \& Hegarty, M. (2002). Multimedia design for communication of dynamic information. International Journal of Human-Computer Studies, 57(4), 279-315.

Palmiter, S., \& Elkerton, J. (1993). Animated demonstrations for learning procedural computerbased tasks. Human-Computer Interaction, 8, 193-216.

Rieber, L. P., Tzeng, S.-C., \& Tribble, K. (2004). Discovery learning, representation, and explanation within a computer-based simulation: finding the right mix. Learning and Instruction, 14(3), 307-323.

Rouet, J.F., Merlet, S., Ros, C., Richard, E., \& Michaux, C. (2002). Effects of animated illustrations on the comprehension of an expository text. Poster Session of EARLI-SIG, "Comprehension of Verbal and Pictorial Information". Poitiers: august 29.

Schwan, S., \& Riempp, R. (2004). The cognitive benefits of interactive videos: learning to tie nautical knots. Learning and Instruction, 14, 293-305.

Schnotz, W., \& Grzondiel, H. (1999). Individual and co-operative learning with interactive animated pictures. European Journal of Psychology of Education, XIV, 245-265. 
Sims, V. K., \& Hegarty, M. (1997). Mental animation in the visuospatial sketchpad: evidence from dual-task studies. Memory and Cognition, 25(3), 321-332.

Tassini, S., \& Bétrancourt, M. (2003). Le contrôle sur l'animation influence-t-elle le niveau d'efficacité cognitive de l'animation. Neuvièmes Journées JETCSIC, 21 juin : Dijon.

Tversky, B., Bauer-Morrison, J., \& Bétrancourt, M. (2002). Animation : can it facilitate ? International Journal of Human-Computer Studies, 57, 247-262. 